\title{
Patient catechism: no prayers please
}

A

British nurse who was suspended without pay after offering to pray for a patient has been reinstated following an investigation by the National Health Service.

Caroline Petrie, a part-time visiting nurse, had been accused of "failing to demonstrate a personal and professional commitment to equality and diversity" as a consequence of a Dec. 15 , 2008 visit to a 79-year-old woman, during which she inquired: "would you like me to pray for you?"

Petrie, a Baptist, says that she did not mention Christianity or any other religion. The patient declined, and later mentioned the offer to another caregiver, which led, in turn, to Petrie's suspension, without pay, 2 days later. She was reinstated in February but the North Somerset Primary Care Trust informed her that she must hereafter inquire whether her patients "have a faith" or any spiritual needs before offering to pray for them. In a statement, the trust said: "It is acceptable to offer spiritual support as part of care when the patient asks for it. But for nurses whose principal role is giving nursing care, the initiative lies with the patient and not with the nurse. Nurses like Caroline do not have to set aside their faith, but personal beliefs and practices should be secondary to the needs and beliefs of the patient and the requirements of professional practice."

Petrie's supervisors reprimanded her last October for offering a prayer card to an elderly patient, who accepted it. After the man's caregiver reported the exchange, Petrie received a written warning and was ordered to attend an equality and diversity workshop.

Dr. Andrew Fergusson of the Christian Medical Fellowship says a "culture of fear" may have resulted in some staff overinterpreting National Health Service guidelines on religion and diversity. A new set of guidelines published Jan. 9 contains no explicit ban on prayer, but warns that religious beliefs should not adversely affect patient care.

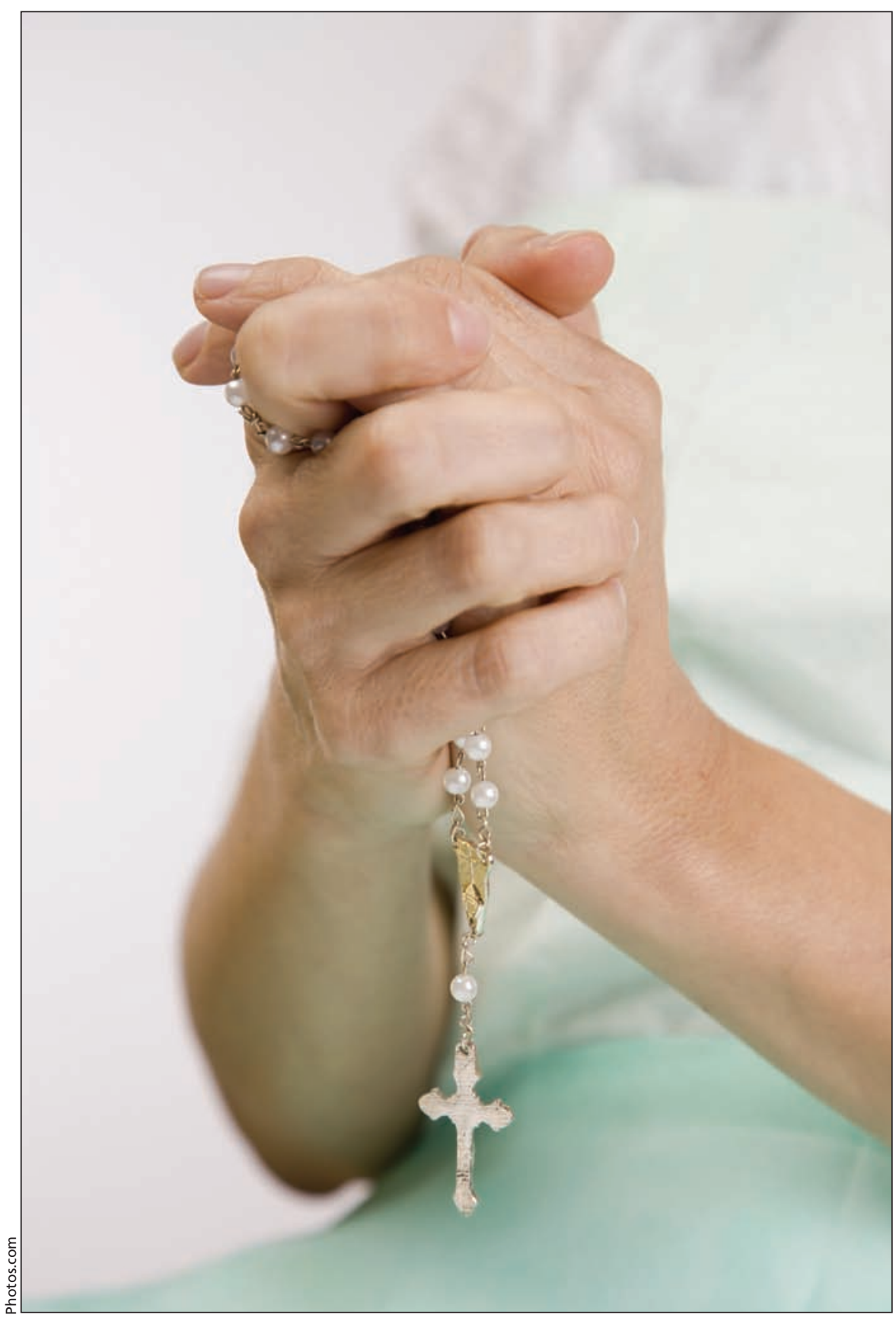

Spiritual support by health care workers is often frowned upon.

Nor should the religious beliefs of patients be allowed to interfere with staffers carrying out their duties.

Fergusson adds that while there's been an improvement in National Health Service chaplain services over the past 2 decades, there also seems to be an increasing intolerance of spiritual matters. - Mary Helen Spooner, London, England

DOI:10.1503/cmaj.090405 